\title{
Die Funktionsprüfung des Herzens in der Praxis des Arztes.
}

Von Prof. H. Rosin in Berlin.

Bis vor nicht langer Zeit gründete sich die Herzdiagnostik im wesentlichen auf die Ergebnisse der physikalischen Untersuchungsmethoden; Horchen und Klopfen, Betrachtung des Aussehens, Fühlen des Pulses, Beobachtung der Atmung waren ausschlaggebend für die Beurteilung von Art und Grad der Erkrankung. Auch heute bleibt diese Methode, verfeinert durch Apparate aller Art, unter denen der Röntgenapparat an der Spitze steht, die wichtigste und ergebnisreichste für Theorie und Praxis. Zweck und Ziel aller physikalischen Untersuchungsmethoden ist in erster Reihe die Feststellung der a na to mis che $\mathbf{n}$ Verhältnisse, also der Größenverhältnisse des Herzens, die Veränderungen an den Klappen, die Weite und die Veränderungen an den großen Gefäßen.

Neuerdings in den letzten Jahrzehnten und in immer stärkerem Grade, ist die Neigung hervorgetreten, der Herzf unktion eine gesteigerte Aufmerksamkeit zuzuwenden. Man darf freilich nicht übersehen, daß auch schon früher, gerade mit Hilfe der physikalischen Untersuchungsmethoden, auch funktionelle Diagnostik getrieben wurde. Besonders die sorgfältige Beobachtung des Pulses führte zu funktionellen Erkenntnissen, und die Kardiographie und Sphygmographie, die in den siebziger und achtziger Jahren des vorigen Jahrhunderts zur höchsten Blüte kamen, bestrebten sich, nicht die anatomischen Verhältnisse, sondern die Leistung des Herzens zur Anschauung zu bringen. Besonders Ottomar Rosenbach wurde nicht müde, auf den Wert der funktionellen Diagnostik hinzuweisen.

Dennoch ist die Neigung funktioneller Betrachtungsweise aus der Verborgenheit einzelner klinischer und wissenschaftlicher Institute auf die allgemeine Denkungsrichtung der im praktischen Leben stehenden Aerzte erst übergegangen, als man erkannt hatte, daß die anatomische Richtung allein nicht befriedigen kann. $\mathrm{Zu}$ oft hatte man gefunden, nicht zum mindesten an der Hand von Röntgenbildern, daß anatomisch abnorm gestaltete, vergrößerte oder auch zu kleine Herzen durchaus ihren Anforderungen genügten und daß umgekehrt grobe Störungen der Herzkraft da vorkamen, wo die physikalische Untersuchung annähernd normale Verhältnisse aufzuweisen schien. O. Rosenbach prägte um 1890 herum das späterhin so oft gebrauchte Wort der Reservekraft des Herzens; auf ihr Fehlen oder Vorhandensein stützt sich die funktionelle Untüchtigkeit oder Leistungsfähigkeit. $O$. Rosenbach folgten bald eine große Zahl von Klinikern und Praktikern, welche den vorgezeigten Weg der funktionellen Betrachtungsweise des Herzens betraten.

Heute kann man eine ganze Reihe von Methoden zusammenstellen, die der Herzfunktion, nicht der Herz. for $\mathrm{m}$ gewidmet sind. Ein Teil davon ist höchst einfach und für die Praxis geeignet, ein anderer Teil beruht auf der Verwendung komplizierter Apparate, die Mühe und Zeit erfordert und die mehr für die Klinik zu brauchen ist. Ich will mich bemühen, im Nächstfolgenden, dem Wunsche der Redaktion entsprechend, die für die Praxis wichtigen Methoden zusammenzustellen.

I. Prüfung der Herzfunktion nach Muskelarbeit durch Zählung der Herzschläge.

Auf physiologische Beobachtungen stützt sich der ebenso bekannte wie brauchbare $\mathrm{K}$ niebeugeversuch zur Prüfung der Herzfunktion. Seine Ausführung ist einfach.

Man stellt bei dem zu Untersuchenden nach längerer Ruhe zunächst im Stehen, am besten auch im Liegen, die Pulszahl fest, läßt zehn ergiebige Kniebeugen machen, bestimmt un- 
mittelbar danach die Pulsfrequenz, wartet fünf Minuten und bestimmt sie aufs neue. Unter normalen Verhältnissen ist $u$ nmittelbar nach dem Kniebeugen die Pulsvermehrung öfter erheblich, zuweilen um die Hälfte höher als vorher, und hin und wieder ist der Puls ganz außerordentlich beschleunigt. Allein bei normaler Herzfunktion muß nach fünf Minuten unter allen Umständen die Ziffer der Pulsfrequenz wieder ges u nke n sein, und zwar fast oder ganz auf die Ruheziffer.

Dieser einfache Versuch ist in der Praxis recht brauchbar. Er stützt sich auf die in der Physiologie gefundenen Tatsachen, welche dieHerzleistung gegenüber Muskelarbeit betreffen. Muskelarbeit bedeutet für das Herz nichts anderes als Vermehrung der Widerstände. Die sich zusammenziehenden Muskeln komprimieren die Gefäße, die in ihnen verlaufen; je mehr Muskeln in Aktion treten, je kräftiger sie arbeiten, einen um so stärkeren Widerstand findet die arterielle Zufuhr in den Gefäßen, die freilich beim Nachlassen der Muskelkontraktion jedesmal wieder in reichem Maße stattfindet; das Blut der Venen wird bei der Kontraktion der Muskeln gleichzeitig herausgedrückt. Das Herz ist genötigt, die partielle Verengerung der Blutbahn durch Mehrarbeit zu überwinden, es geschieht dies mit verstärkter Aktion und durch vermehrte Frequenz. So nimmt schon beim einfachen Stehen gegenüber dem Liegen die Pulszahl (und der Druck) zu, da eine Reihe von Muskeln zur Erhaltung des Gleichgewichts beim Stehen in Aktioñ treten; das Herz arbeitet stärker und frequenter; man sagt, das Schlagvolumen steigert sich. Bei dieser Mehrleistung bleibt das Gefäßnervensystem sicher nicht untätig, es reguliert die Widerstände in den Muskeln durch Erweiterung anderer Gefäßgebiete vom Zentralorgan aus, wobei verschiedene Nerven, unter anderen auch der N. depressor, wirkungsvoll eingreifen. Leider ist diese funktionelle Tätig keit der Gefäßnerven im wesentlichen noch unbekannt. Auf das Verhalten des Herzens aber, das man kennt, stützt sich obiger Kniebeugeversuch. Er berüicksichtigt nur die Pulsbeschleunigung, nicht die Blutdrucksteigerung.

In pathologischen Fällen bleibt auch fünf Minuten nach dem Versuche die Pulsfrequenz bedeutend gesteigert. Man muß freilich hier wie bei allen pathologischen Vorkommnissen mit Uebergängen rechnen. Eine geringe Vermehrung um wenige Schläge wird bei gewissen Individuen, z. B. bei nervösen, erregten Menschen und besonders bei solchen, bei denen die Pulsfrequenz vorher etwas beschleunigt war, nicht gleich als krankhaft anzusehen sein. Wenn aber der Patient vor dem Versuche z. B. 80 Schläge, nach dem Versuche längere Zeit 100 Schläge zeigt, so ist mit Recht eine Funktionsuntüchtigkeit des Herzmuskels zu vermuten.

Die Ziffer 10 als Zahl der vorzunehmenden Kniebeugen hat sich an der Hand zahlreicher Versuche bewährt. In schweren Fällen von Herzerkrankungen, bei denen man freilich die Methode gewöhnlich nicht braucht, muß man vorsichtig sein und sich höchstens mit der Hälfte begnügen. Daß anderseits noch stärkere Herzarbeit eine länger andauernde Vermehrung der Pulsfreqenz erzeugen würde, ergibt sich schon aus der einfachen Betrachtung des Verhaltens der Pulsfrequenz nach größeren körperlichen Anstrengungen, z. B. nach Rudern oder Bergsteigen; hier tritt auch beim Gesunden, individuell ganz verschieden, eine starke Steigerung der Pulsfrequenz ein, die nicht selten das Doppelte des Ruhezustandes übertrifft, und ebenso bedarf es längere Zeit, oft eine Stunde und mehr, der Ruhe, bis der ursprüngliche Zustand in der Pulsziffer erreicht ist. Funktionelle Untüchtigkeit zeigt sich hier bei starker Muskelanstrengung erst dann, wenn auch nach Stunden die Pulsziffer hoch bleibt. Der tüchtige Sportsmann wird nicht daran erkannt, daß während der Sportsübung seine Pulsziffer nicht allzusehr in die Höhe geht, sondern viel mehr daran, daß sie rasch in der Ruhe wieder normal wird.

Der Versuch berücksichtigt nur den einen Vorgang bei der Muskelarbeit, die Pulsbeschleunigung, das Verhalten des Blutdrucks und der Atmung wird vernachlässigt. Es sei hervorgehoben, daß über die physiologischen Ursachen der Vermehrung der Herzschläge trotz der Einfachheit des Versuches absolute Klarheit noch nicht geschaffen ist. Man vermutet Erregung des Herznervenzentrums (in gleicher Weise wie die Erregung des Zentrums der Vasomotoren und der Atmung), vielleicht durch die Ermüdungsstoffe des Muskels (s. unten Weber) oder durch die vermehrte Bildung von Kohlensäure. Nach eingetretener Muskelruhe hört die zentralerregte Herzbeschleunigung alsbald wieder auf; sie kehrt (wie auch Blutdruck sowie Atembeschleunigung) zum Ruhestand zurück. In pathologischen Fällen wird die längere Dauer der erhöhten Pulsfrequenz durch Retention von Ermüdungsstoffen und Kohlensäure als Folge ungenügender Zirkulation erklärt.

Durch den Kniebeugeversuch kann man sich in der Eile der Praxis sehr gut einen gewissen Einblick in die Herzfunktion verschaffen. Apparate aller Art bieten keinen Vorteil und erschweren die Methodik. Erwähnt sei deshalb nur, daß man $a b$ und zu dem Ergostaten als Prüfer der Herzfunktion begegnet, einem von Gärtner für Muskelübungen konstruierten Apparate, der (nach Gräupner) eine genauere Dosierung der Muskelarbeit gestatten soll.

\section{Prüfung der Herzfunktion mittels Blutdruckmessung.}

Der gesunde Erwachsene hat stets einen bestimmten, manometrisch meßbaren Blutdruck in der A. brachialis, der in der Norm nur in engen Grenzen variiert.

Der für die Blutdruckmessung in der Praxis allgemein eingeführte Apparat ist derjenige von Riva-Rocci mit der Recklinghausenschen Manschette, einer etwas breiteren, als sie ursprünglich von Riva-Rocci angegeben war. Der Apparat ist etwas schwer transportabel; doch hat Vollhardt eine leicht tragbare Form hergestellt, die freilich wegen einer gewissen Zerbrechlichkeit etwas Geschicklichkeit erfordert. Beide Apparate insgesamt sind nicht teuer. Der Blutdruckmesser darf in der Praxis eines jeden Arztes nicht mehr fehlen.

Mittels des Apparats kann man die Kraft, mit der der linke Ventrikel in der A. brachialis arbeitet, einigermaßen beurteilen; die nicht zu beseitigende Fehlerquelle, daß man den aktiven Druck der Gefäßwand auf die Blutsäule, d. h. den Einfluß der Vasomotoren mitmißt, spielt trotz ihrer theoretischen Bedeutung in der Praxis keine absolut störende Rolle. Man bestimmt mittels der Methode zwei Momente des Blutdrucks im Gefäßsystem: 1. Den maximalen Blutdruck, wie er durch die Systole der linken Kammer (in Verbindung mit dem Vasomotorendruck) in der A. brachialis erzeugt wird. Er wird am Manometer des Apparates abgelesen mittels Palpation in dem Momente, in welchem der Radialpuls eben gänzlich verschwindet, oder, mittels Auskultation, dann, wenn ein vorher in der Ellenbogenbeuge hörbarer Brachialton verschwindet, resp. nach Erzeugung eines Ueberdruckes und dessen allmählicher Verringerung wieder auftritt. 2. Den minimalen Blutdruck der dann abgelesen wird, wenn der Radialpuls, vorher durch die Kompression der Manschette etwas verkleinert, wieder seine volle Höhe erreicht hat. Diesen Punkt kann man mittels Palpation schwer erkennen, dagegen sehr gut durch Auskultation der Brachialis in der Ellenbogenbeuge, da der Brachialton, der bei mäBiger Kompression stets vorhanden ist, im Momente des Auftretens des vollen Pulses verschwindet.

Man hat die Differenz zwischen maximalem Blutdruck, der auch systolischer Blutdruck genannt wird, und dem minimalen, auch diastolisch genannten, mit dem Ausdrucke Pulsdruck bezeichnet und vorübergehend Hoffnungen daran geknüpft, durch die Bestimmung dieses Pulsdruckes ein Maß für die gesamte Herzleistung zu gewinnen. Doch ist die Erkenntnis, daß, wie oben schon erwähnt, der Druck in einer Arterie nicht lediglich von der Herzkraft abhängig ist, sondern auch von den überaus kräftig wirkenden Vasomotoren, welch letztere man vom Herzdruck leider nicht trennen kann, die Veranlassung gewesen, von jenem Standpunkte abzukommen. Zurückgeblieben ist aber doch für die Beurteilung der Herzfunktion die Messung des systolischen, maximalen Blutdrucks; diese Messung ist eine für die Praxis überaus wichtige Methode. Der Erwachsene mit normaler Herztätigkeit hat, wie schon erwähnt, einen ganz bestimmten, nicht sehr schwankenden maximalen Blutdruck. Er beträgt durchschnittlich $120 \mathrm{~mm} \mathrm{Hg}$, kann' aber zwischen 110 und 130 schwanken: schwächlichere $]$ und jüngere Individuen haben einen niedrigeren als kräftigere und ältere.

Die Messung des maximalen Blutdrucks kann als Maß verwendet werden für die Tüchtigkeit der Herzfunktion. Jede 
gesteigerte Herzarbeit, wie sie durch Muskeltätigkeit bedingt wird, führt zur Steigerung des Blutdrucks, beim Gesunden aber nur vorübergehend. Läßt man den Patienten zehn Kniebeugen machen, so soll nicht nur die Pulsfrequenz, sondern auch der Blutdruck wieder auf den Stand gesunken sein, wie er vor der Arbeitsleistung war. Man kann dies leicht feststellen, wenn man den Patienten, mit der Blutdruckmanschette bewaffnet, zehn Kniebeugen machen läßt und nach fünf Minuten abliest.

Aus den Abweichungen von diesen Normalziffern kann man nun Schlüsse auf die Herzfunktion machen. Ist der systolische Druck in der A. brachialis zu niedrig, so liegt eine Schwäche der Herzkraft vor. Bei dem Drucke z. B. von $98 \mathrm{~m} \mathrm{Hg}$ kann man eine Schwäche der Herzmuskulatur ohne weiteres annehmen, auch wenn man theoretisch erwägt, daß der Tonus der Gefäße, die Vasomotoren einen gewissen Anteil am Zustandekommen des Blutdrucks haben. Die klinische Beobachtung bestätigt auch stets, da $\beta$ der Unterdruck im wesentlichen auf Schwäche des Herzmuskels zurückzuführen ist.

Umgekehrt hat auch der gesteigerte Blutdruck eine hohe Bedeutung für das Verständnis der Herzleistung. Er gibt meist an, daß die Herzarbeit abnorm gesteigert ist, gesteigert durch allerlei Ursachen, die in der Regel auch zu anatomischen Steigerungen der Größe und Ausdehnung der linken Kammer führen.

Die allerhöchsten Ziffern des maximalen Blut. drucks zeigt stets die Schrumpfniere, die genuine sowohl wie die arteriosklerotische, letztere oft oh ne $\mathrm{Ei}$ weißa us scheidung. Der svstolische Blutdruck liegt hier über 200, und es kommen nicht selten Fälle vor, in denen die gewöhnlichen Manometer, die bis 240 graduiert sind, für ihn nicht ausreichen. Eiweißausscheidung ohne Blutdrucksteigerung gehört nie der Schrumpfniere an. Etwas weniger hoch steht er bei Arterioskle rose ohne Nierenkomplikation. Hier sind Steigerungen bis $190 \mathrm{zu}$ beobachten. Die beginnende Arteriosklerose (Präsklerose) oder vorübergehende Blutdrucksteigerungen zeigen niedrigere Werte von 140--150. Doch gibt es auch lokale Arteriosklerose ohne Blutdrucksteigerung (eventuell Arteriosklerose der Koronararterien, der Aorta, der Hirnarterien). Unter den Herzfehlern lassen die meisten nur dann Blutdrucksteigerungen mäßigen Grades erkennen, wenn sie Hypertrophien der linken Kammer haben und dabei gut kompensiert sind, so z. B. die Mitralfehler, die eine Steigerung bis etwa 150 aufweisen können. Auch kann hier der Blutdruck noch normal sein, je nach dem Verhalten der Hypertrophie und des Tonus der Gefäße. Ein Sinken des Blutdrucks bei Mitralfehlern auf seine niedrigste normale Stufe (110) oder darunter ist schon als Herzschwäche anzusehen. Bei derAortenkla p penins uffizienz istder Blutdruck, guteKompensation vorausgesetzt, fast immer erheblich gesteigert. Die meist gleichzeitig vorhandene Arteriosklerose, ebenso die so häufige Nephritis ist oft dabei beteiligt. Aber auch in reinen endokarditischen Fällen steht der Blutdruck oft hoch infolge der Hypertrophie der linken Kammer zwischen 150 und 180. Charakteristisch für die Aorteninsuffizienz ist daß bei Dekompensation der Blutdruck meist nicht zur Norm oder noch tiefer sinkt, wenigstens bei den Fällen, die mit Arteriosklerose und Schrumpfniere kompliziert sind. Trotz deutlicher Zeichen der Herzinsuffizienz bleibt der Hochdruck bis ans Lebensende bestehen. Kein Zweifel, daß hier die Spannung und der Widerstand im arteriellen Gefäßsystem die Ursache dieser Drucksteigerung ist, nicht der Herzmuskel, dessen Kraft erlahmt ist; die Vasomotoren haben hier also einen Hauptanteil daran. Wir sehen in solchen Fällen besonders, daß die Ziffern des Blutdrucks sich als die Summe derjenigen der Herzleistung und des Gefäßdruckes enthüllen. Wir erkennen auch ihr gegenseitiges Ineinandergreifen beim Gesunden in der Weise, daß der Blutdruck stets nur in engen Grenzen schwankt und sich im allgemeinen gleich bleibt. Wir müssen aber vorläufig mangels Einsicht in die Verhältnisse des Gefäßdruckes die Blutdrucksenkung als Herzschwäche deuten, die Blutdrucksteigerung als gesteigerte Herzarbeit, und können dies auch in der Praxis durchführen.

\section{Kombinierte Verfahren.}

a) Es lag nahe, die Herztüchtigkeit in kombinierter. Weise mittels Pulszählung und Blutdruckmessung nach einer bestimmten Arbeitsleistung zu prüfen. Schrum pf hat kürzlich eine derartige, für den praktischen Arzt brauch bare Methodik geschaffen ${ }^{1}$ ). Im Liegen werden diastolischer und systolischer Druck sowie Pulszahl bestimmt. Die Druck bestimmung erfolgt mittels der auskultatorischen Methode (s. oben), also mittels Auskultation der A. brachialis in der Ellenbogenbeuge. Wegen psychischer Momente sind zwei bis drei Messungen nötig. Der diastolische Blutdruck wird mit zunehmendem, der systolische mit abnehmendem Druck bestimmt. Man läßt dann den Patienten langsam aufstehen, wartet zwei Minuten, um den Einfluß der Bewe ung auszuschalten, und mißt dann wieder Druck und Puls. Dann läßt man tiefe Kniebeugen ausführen, 5-20 je nach dem Zustande des Herzens. Der an das Manometer angeschlossene Manschettenschlauch muß schnell abnehmbar und wieder aufziehbar sein, damit alle Ablesungen schnell erfolgen können; andernfalls muß man das Manometer bei allen Bewegungen des Patienten mit der Hand mitbewegen. Nach den Kniebeugen werden rasch im Sitzen Puls- und Blutdruckbestimmungen vorgenommen und diese noch viermal in Minutenabstand wiederholt. Dann legt man den Patienten wieder hin und mißt nach der fünften Minute zum letzten Male Blutdruck und Pulszahl. Man kann zum Schlusse der Untersuchung noch die Zahl der Atemzüge sich notieren. Die erhaltenen Ziffern werden auf einer Kurve aufgezeichnet. Als Apparatur für den ganzen Versuch bedarf man nur des oben geschilderten Blutdruckapparates sowie einer Vorrichtung, um die Luft aus der Manschette besonders schnell herauslassen zu können (Quetschhahn am vierten Ast eines + -Rohres).

Diese Methode der Prüfung der Herzfunktion Schrumpfs zeigt in einfacher Weise einen Ma Bstab der Herztüchtigkeit an, der vollauf für die Bedürfnisse des praktischen Arztes genügt. Unter normalen Verhältnissen zeigt sich das Bild folgendermaßen: Beim Uebergang von der liegenden in die stehende Stellung erfolgt eine Zunahme um drei bis sechs Schläge in der Minute, nach der Arbeit um 10-20 Schläge. In längstens vier Minuten ist die Pulszahl zur Norm zurückgekehrt. Die Blutdruckbestimmung ist ähnlich: der maximale (systolische) Druck nimmt beim Uebergange von der liegenden in die stehende Stellung um höchstens $10 \mathrm{~mm}$, nach der Arbeit um $20-40 \mathrm{~mm}$ zu. In längstens vier Minuten ist die Norm wieder erreicht. Der minimale Druck (diastolische) nimmt im ganzen nicht über $10 \mathrm{~mm} \mathrm{zu}$, in drei bis vier Minuten ist er zur Norm zurückgekehrt. Es zeigt sich also am normalen Herzmuskel, daß er über genügend Reservekraft verfügt, um sie bei vermehrter Inanspruchnahme des Herzens anzuwenden, aber nur so lange, als die vermehrte Anstrengung sie erfordert. Die Reservekraft tritt aber sofort wieder außer Funktion, wenn der Ruhestand wieder eingetreten ist, weil dann die ursprüngliche Herzkraft vollkommen ausreicht.

Ganz anders verhält sich die Puls- und Druckkurve nach Schrumpf bei Störungen der Herzmuskeltätigkeit. Bei schwerer Herzerkrankung ist selbstverständlich diese Methodik der Funktionsprüfung unnötig. Nur bei leichten, nicht ganz klaren Symptomen von Inkompensation und zur Feststellung einer nur vermuteten Herzmuskelsch wäche ist sie von äußerstem Nutzen.

So zeigt sich Herzschwäche, bedingt durch allerlei Ursachen, in auffallender Zunahme des Pulses beim Uebergang von der liegenden in die stehende Stellung $(10-20$ Schläge und mehr), und mehr noch nach der Muskelarbeit (weitere 20-30 Schläge und darüber), außerdem findet eine langsame Rückkehr zur Norm oft erst nach zehn Minuten und länger statt. Der Blutdruck nimmt dafür nicht genügend zu beim Uebergang in die stehende Stellung und auch nach der Arbeit nicht beträchtlich genug (höchstens $10 \mathrm{~mm}$ ). Diese zu geringe Steigerung bleibt dann während der ganzen fünf Minuten bestehen. Weitere Einzelheiten der Schrumpfschen Methodik bei verschiedenen Herzkrankheiten werden sich dem Praktiker bei ihrer Anwendung von selbst ergeben.

b) Vor fast zehn Jahren hat Katzenstei ${ }^{2}$ ) ein einfaches und praktisch sehr brauchbares Verfahren zur Prüfung der Herzkraft eingeführt, das ebenfalls empfohlen werden kann. Auch er prüft wie Schrumpf Blutdruck und Pulszahl am Arm vor und

1) Med. Klin. 1916 Nr. 25. - 2) D. m. W. 1904 Iir. 22 u. 23. 
nach erhöhter dem Herzen auferlegter Leistung. Diese erhöhte Leistung wird hier durch Erzeugung eines künstlichen Widerstands im großen Kreislauf bewirkt. Es werden nämlich beide Aa.femorales a $m$ Poupartschen Bande mit de $m$ Finger komprimiert, die Kompression erfolgt allmählich so intensiv, daß der Puls darunter verschwindet, was der palpierende Nachbarfinger festzustellen hat. Steigerung des Blutdrucks bei Verminderung der Pulszahl nach der Kompression läBt auf ein normales Herz schließen. Abnorme Steigerung des Druckes zeigt sich bei Arteriosklerose und Herzhypertrophie. Keine Blutdruckerhöhung und keine Aenderung des Pulses spricht für geringe Schädigung des Herzmuskels, die größer wird, je mehr die Pulszahl zunimmt. Verminderung des Blutdruckes bei Erhöhung der Pulszahl läßt auf eine schwere Insuffizienz schließen.

Die Ergebnisse dieses Versuches unterscheiden sich erheblich vom Kniebeugeversuch. Während bei diesem die Pulsfrequenz vermehrt ist, ist bei dem Katzensteinschen Versuch unter funktionell normalen Verhältnissen eine Pulsverlangsamung da. Der Unterschied liegt offenbar in der Art der Anordnung. In dem einen Falle besteht Muskelarbeit, in dem anderen Muskelruhe, aber Ausschaltung eines größeren Abschnittes des arteriellen Kreislaufs. Offenbar reagiert Herz und GefäBsystem auf diese beiden Widerstandsarten prinzipiell verschieden; wir haben allen Grund anzunehmen, daß dabei Stoffwechselprodukte des Muskels eine Rolle spielen, die bei dem Kniebeugeversuch sich bilden, bei dem Kompressionsversuch der Femoralis aber fehlen.

c) Ein drittes für die Praxis brauchbares Verfahren hat Rehfisch ausgearbeitet. ${ }^{1}$ ) Während die bisher genannten Methoden lediglich die Leistungskraft des linken Ventrikels priifen, kann die seinige auf beide Ventrikel verwendet werden. Bekanntlich ist der $z$ weite Aortenton lauter als der zweite Pulmonalton, wenigstens unter normalen Verhältnissen, denn der Druck im arteriellen Körperkreislauf ist etwa um ein Drittel höher als im Lungenkreislauf. Bei gesundem Herzen muß nach Arbeitsleistung das Verhältnis das gleiche bleiben. Bei Schwächung des linken Ventrikels wird der zweite Aortenton herabgesetzt und dẹr zweite Pulmonalton dafür relativ stärker. Wird auch der zweite Pulmonalton in gleicher Weise schwächer, so ist auch die Muskulatur des rechten Herzens untüchtig. Die Methode ist einfach und brauchbar, sie hat aber zur Voraussetzung, daß keine Arteriosklerose vorliegt, denn in diesem Falle ist bekanntlich der zweite Aortenton verstärkt und klingend aus Gründen der Einbuße der Elastizität und der oft vorhandenen Drucksteigerung trotz oft insuffizienten Herzens.

\section{Die Webersche Methode und andere Verfahren.}

Wir kommen nunmehr zu Methoden der Funktionsprüfung des Herzens und der Gefäße, die zum Teil zwar für die Praxis sehr brauchbar, aber durch Beschaffung und Anwendung eines umfangreicheren Apparates kostspielig sind und vor allem Zeit gebrauchen und daher wohl nur in den Händen des praktischen Spezialisten Verwendung finden können.

Eine verhältnismäßig einfache Methode, die schon Erfolge erzielt hat und noch weitere verspricht, ist diejenige von Ernst Weber. ${ }^{2}$ ) Sie stützt sich auf die alte, in der Praxis niemals eingebürgerte Methode der Plethysmographie. Es wird das Armvolumen plethysmographisch aufgeschrieben während der Ausführung einer kräftigen, aber auf bestimmte Weise völlig lokalisierten Muskelarbeit eines Fußes (Beugen und Strecken) bei einer Dauer von 10-15 Sekunden unter gleichzeitiger Registrierung der Atembewegungen. Weber hat an Tausenden von Gesunden festgestellt, $\mathrm{daB}$ bei dieser Arbeit eine Vermehrung der Blutfülle sämtlicher äuBerer Körperteile eintritt a ußer denen des Kopfes, neben gleichzeitiger Abnahme der Blutfülle der Bauchorgane und Zunahme der des Gehirns. Der Zweck dieses Vorgangs ist offenbar der, die Muskeln gründlich zu durchbluten, damit ihnen reichlich Sauerstoff zuzuführen und zugleich die Ermüdungsstoffe zu entfernen, wodurch die Erschöpfung länger hinausgeschoben wird. Weber hat dann weiter gefunden, daß bei starker Ermüdung durch andauernde Muskelarbeit eine Umkehr stattfindet, auch wenn die Muskelarbeit nur lokalisiert ist. Dann verengen sich alle äußeren Blutgefäße des Körpers. Nach längerer Ruhezeit, oft nach etwa zwei Stunden, tritt das normale Verhältnis wieder ein. Die Ursache dieser konträren Reaktion ist nach Weber auf die Vermehrung der Weichhardtschen „Ermüdungsstoffe " zurückzuführen, welche den zentralen Innervationsmechanismus der Gefäße in entgegengesetztem Sinne beeinflussen; die allzusehr vermehrten Ermüdungsstoffe kehren die Wirkung der Muskelarbeit auf die Blutverteilung bei Ermüdung um. W eber hat nun ferner gezeigt, daß diese sogenannte umgekehrte Gefäßreaktion bei Muskelarbeit nicht nur bei Uebermüdung sich findet, sondern auch bei verschiedenen Arten von Erkrankungen. Hierher gehören vor allem die Vergiftungen; bei akuten Vergiftungen ist die Schädigung des vasomotorischen Systems plethysmographisch schon am folgenden Tage festzustellen.

Vor allem aber fand sich bei schwerer Herzkrankheit dieselbe umgekehrte Reaktion. Es dehnen sich die peripherischen Gefäße in den Muskeln nicht aus, die inneren Organe entbluten nicht, die Gefäße des Gesichts verengen sich nicht, das Gehirn wird nicht blutreicher. Der Plethysmograph zeigt also eine Verminderung des Armvolumens an, die Brust- und Bauchhöhle bleibt blutreich usw. DieseVerhältnisse bei Schwächezuständen des Herzmuskels im Verlauf aller möglichen Herzkrankheiten erklären sich in ähnlicher Weise wie bei übermäßiger Muskelermüdung. Denn bei Herzkrankheiten ist es die durch die mangelnde Zirkulation vermehrt auftretende Kohlensäure : in Verbindung mit der mangelhaften Ausscheidung von Stoffen ungenügender Sauerstoffverbrennung, von Schlacken, im Blute, welche die perverse Reaktion (ähnlich wie die Ermüdungsstoffe) erzeugt.

Damit hat Weber eine Methodik geschaffen, welche gestattet, unzulängliche Zirkulation, durch unzulängliche Herzarbeit bedingt, frühzeitig zu erkennen. Mit ihr ist eine Beurteilung der Leistungskraft des Herzens ermöglicht: Will man wissen, ob der Herzmuskel funktionell tüchtig ist, so läBt man mit dem Plethysmographen eine Kurve aufschreiben, sie muß beim Webersche $n$ Versuch ansteigen, d. h. das Armvolumen muß sich füllen, sinkt die Kurve, so tritt die falsche Reaktion des geschwächten Herzmuskels ein. Eine solch negative Reaktion zeigt sich auch nach Herzmuskelschwäche im Anschlusse an durchgemachte schwere Infektionskrankheiten. Sie entscheidet, $\mathrm{ob}$ ein für schwach angenommenes Herz leistungsfähig ist, körperliche Anstrengungen zu ertragen, sie entscheidet ferner, $o b$ ein Herz nur nervös oder muskelschwach ist, ob ein zu kleines Herz noch tüchtig ist, ob ein durch Ueberanstrengungen zeitweise geschwächtes Herz wieder leistungsfähig geworden ist. Wichtig ist, die Beobachtung, daß auch bei vorhandener normaler Reaktion hin und wieder die Rückkehr der angestiegenen Kurve zur Norm sehr langsam erfolgt, ein Zeichen geringerer, aber noch vorhandener Muskelschwäche, oder daß sie nach dem Abfall längere Zeit abnorm tief liegt, ebenfalls ein Zeichen muskulärer Schwäche. Manchmal, besonders bei arteriosklerotischer Aortenerweiterung, ist der Anstieg schräg und langsam.

Zum Schlusse sei eine Anzahl von Apparaten nur der Vollständigkeit halber erwähnt, die aber für die Praxis, auch für die spezialistische, nur einen bedingten Wert haben, dafür aber klinisch-wissenschaftlichen Untersuchungen bestens dienen können.

Genannt seien hier zunächst das Sahlische Sphyg mobolometer, ${ }^{1}$ ) das Christensche Energometer ${ }^{2}$ ) und der Straussche Turgotonograph. Genannt seien ferner die Sphymographen verschiedener Konstruktion; sie bleiben ebenfalls wesentlich der Klinik vorbehalten, schon darum, weil sie weniger der Erforschung der Tüchtigkeit des Herzens dienen als der Art der Herzbewegung, der Beziehung des Herzens zu den Gefäßen und der Beziehungen der Vorhöfe zu den Ventrikeln.

Der Elektrokardiograph, von Einthoven eingeführt, von Edelmann, Nicolai und Siemens \& Halske in verschiedenen Formen konstruiert, sehr teuer im Preise, nicht leicht und nur zeitraubend $\mathrm{zu}$ bedienen, wird einst dem praktischen Herzspezialisten nicht fehlen dürfen, wenn seine praktische Verwertung besser ausgebaut sein wird. Schon jetzt zeigt er dem Forscher wichtige funktionelle Tatsachen an, wenn auch sein

(1)

1) B. kl. W. 1915 Nr. 48. - $\left.{ }^{2}\right)$ Zschr. f. exper. Path. u. Ther. 18.

1) D. m. W. 1907 Nr. 16 u. 17. - 2) Zschr. f. klin. Med. 47. 
Hauptwert in der Diagnose und Prognose liegt. Seine Anwendung betrifft hauptsächlich das Gebiet der Störungen des Herzmuskels. in Rücksicht auf den Ablauf des ihn treffenden Kontraktionsreizes. Wir wissen, daß der normale Herzreiz durch das Hissche Bündel läuft, von der Einmündung der V. cava bis zur Herzspitze. Der Elektrokardiograph mißt nun die elektrischen Aktionsströme, die bei den Kontraktionen der Herzabschnitte auf noch nicht in einzelnen geklärte Weise im sich kontrahierenden Muskel aktiv entstehen. Man leitet sie aus den oberen Extremitäten durch Elektrodenwannen in den Elektrokardiographen ab. Sie geben eine Kurve, deren Form unter normalen Verhältnissen eine ganz bestimmte ist, die erste Erhebung (die A-Zacke nach Kraus) entspricht der Vorhofskontraktion, die zweite (die J-Zacke) der Ventrikelkontraktion, eine drifte, letzte, Finalzacke (die F-Zacke) ist in ihrer Deutung noch nicht geklärt. Die Störungen der Kontraktilität des Herzmuskels zeigen sich an der Zacke $\mathbf{A}$ und J. Ihre hier im einzelnen nicht zu besprechenden Unregelmäßigkeiten, die Umkehr der J-Zacke, das Fehlen der A-Zacke oder F-Zacke, dienen oft als wichtige Beweismittel gestörter Herzfunktion.

Die im Voranstehenden geschilderte Methodik der Prüfung der Herzarbeit ist aus dem Wunsche der Forscher hervorgegangen, möglichst fr ühzeitig ein Urteil über die Herzkraft zu gewinnen. Die gröbere Herabsetzung der Leistungsfähigkeit des Herzens ist leicht und sicher mit älteren Methoden zu erkennen, sie werden aber durch die neueren ergänzt und vervollkommnet. 